Published in final edited form as:

Exp Brain Res. 2012 August ; 220(3-4): 251-259. doi:10.1007/s00221-012-3134-4.

\title{
The spatial range of contour integration deficits in schizophrenia
}

\author{
Brian P. Keane, \\ Division of Schizophrenia Research, University Behavioral HealthCare, University of Medicine \\ and Dentistry of New Jersey, 151 Centennial Ave, Piscataway, NJ 08854, USA \\ Department of Psychiatry, UMDNJ—Robert Wood Johnson Medical School, 671 Hoes Lane, \\ Piscataway, NJ 08854, USA \\ Center for Cognitive Science, Rutgers University, New Brunswick, 152 Frelinghuysen Road, \\ Piscataway, NJ 08854, USA
}

\section{Steven M. Silverstein,}

Division of Schizophrenia Research, University Behavioral HealthCare, University of Medicine and Dentistry of New Jersey, 151 Centennial Ave, Piscataway, NJ 08854, USA

Department of Psychiatry, UMDNJ—Robert Wood Johnson Medical School, 671 Hoes Lane, Piscataway, NJ 08854, USA

\section{Deanna M. Barch,}

Departments of Psychology, Psychiatry, and Radiology, Washington University in St. Louis, One Brookings Drive, Box 1125, St. Louis, MO 63130, USA

\section{Cameron S. Carter,}

University of California, Davis, 4701 X Street, Sacramento, CA 95817, USA

\author{
James M. Gold, \\ Maryland Psychiatric Research Center, University of Maryland School of Medicine, P.O. Box \\ 21247, Baltimore, MD 21228, USA
}

\section{llona Kovács,}

Department of Cognitive Science, Budapest University of Technology and Economics, 1 Egry J. u., Bldg T., Budapest 1111, Hungary

\author{
Angus W. MacDonald III, \\ Department of Psychology, University of Minnesota, N426 Elliott Hall, 75 E. River Rd., \\ Minneapolis, MN 55455, USA
}

\author{
J. Daniel Ragland, and \\ University of California, Davis, 4701 X Street, Sacramento, CA 95817, USA \\ Milton E. Strauss \\ Department of Psychological Sciences, Case Western Reserve University, 10900 Euclid Avenue, \\ Cleveland, $\mathrm{OH}$ 44106-7123, USA
}

Brian P. Keane: brian.keane@gmail.com

\begin{abstract}
Contour integration (CI) refers to the process that represents spatially separated elements as a unified edge or closed shape. Schizophrenia is a psychiatric disorder characterized by symptoms such as hallucinations, delusions, disorganized thinking, inappropriate affect, and social
\end{abstract}


withdrawal. Persons with schizophrenia are impaired at CI, but the specific mechanisms underlying the deficit are still not clear. Here, we explored the hypothesis that poor patient performance owes to reduced feedback or impaired longer-range lateral connectivity within early visual cortex-functionally similar to that found in 5- to 6-year old children. This hypothesis predicts that as target element spacing increases from .7 to $1.4^{\circ}$ of visual angle, patient impairments will become more pronounced. As a test of the prediction, 25 healthy controls and 36 clinically stable, asymptomatic persons with schizophrenia completed a CI task that involved determining whether a subset of Gabor elements formed a leftward or rightward pointing shape. Adjacent shape elements were spaced at either .7 or $1.4^{\circ}$ of visual angle. Difficulty in each spacing condition depended on the number of noise elements present. Patients performed worse than controls overall, both groups performed worse with the larger spacing, and the magnitude of the between-group difference was not amplified at the larger spacing. These results show that CI deficits in schizophrenia cannot be explained in terms of a reduced spatial range of integration, at least not when the shape elements are spaced within $1.5^{\circ}$. Later-developing, low-level integrative mechanisms of lateral connectivity and feedback appear not to be differentially impaired in the illness.

\section{Keywords}

Schizophrenia; Contour integration; Visual integration; Spatial range; Perceptual organization; Grouping

\section{Introduction}

Schizophrenia is a devastating psychiatric disorder that is characterized by hallucinations, delusions, disorganized thinking, flat or inappropriate affect, social withdrawal, and a decline in role functioning. Converging evidence indicates that schizophrenia disrupts not only higher-level cognition but also the entirety of mental life, including sensation and perception (Silverstein and Keane 2011b; Green et al. 2011; Javitt 2009). One kind of processing specifically affected is perceptual organization, which refers to the ability to transform a spatiotemporally disjointed retinal image into a veridical representation of the structure and coherency of the external world. Perceptual organization dysfunction in schizophrenia has been demonstrated in over 50 studies (Silverstein and Keane 2011a; Uhlhaas and Silverstein 2005), and examples include: reduced contrast enhancement between collinear elements (Must et al. 2004); weakened shape-based and location-based grouping (Place and Gilmore 1980; Silverstein et al. 1996); and lessened susceptibility to contextually induced size illusions (Uhlhaas et al. 2006), among others. Such abnormalities are likely not a function of psychiatric medication, and typically cannot be attributed to generalized deficits (Silverstein and Keane 2011a; Knight and Silverstein 2001).

\section{Contour integration and schizophrenia}

The form of perceptual organization that is of greatest interest in the present paper is contour integration (hereafter CI; Field et al. 1993). When orientated elements form a collinear (or co-circular) contour, and when those elements are embedded in a field of similar but randomly oriented elements, the target contour pops-out of the array. The ability strongly depends on low-level stimulus factors such as the relative orientation, spatial separation, and alignment of the target elements (Braun 1999); Field et al. 1993); and may also depend on the inhibition of spurious connections made between adjacent noise elements ( $\mathrm{Li}$ et al. 2006). Monkeys can integrate contours with presentation times as short as $30 \mathrm{~ms}$, suggesting that the capacity is phylogenetically primitive and physiologically early (Mandon and Kreiter 2005). CI tasks have provided insights into a range of issues in visual neuroscience, 
including the effects of aging (Del Viva and Agostini 2007), development (Kovács et al. 1999), and amblyopia (Chandna et al. 2001) on early- to mid-level vision.

The anatomical substrate of $\mathrm{CI}$ is furnished partly by long-range horizontal connections between orientation-tuned spatial frequency detectors in early visual cortex (Kapadia et al. 1995; Li and Gilbert 2002). These axonal connections are excitatory and span coaxial pyramidal cells that are separated by up to $2^{\circ}$ of visual angle (or about $3 \mathrm{~mm}$ of cortex at eccentricities of 4-6 $6^{\circ}$ in V1 (Dresp and Grossberg 1997; Grossberg and Raizada 2000; Kapadia et al. 1995; Loffler 2008; Stettler et al. 2002; Mandon and Kreiter 2005). Feedback from higher cortical areas is also important for mediating top-down influence, especially for more distantly separated elements (Angelucci et al. 2002; Zhang and von der Heydt 2010; Ciaramelli et al. 2007). When elements are spaced within $2^{\circ}$, modulatory feedback downstream from V2 probably does not play an important role because: (1) persons with frontal and temporal lobe lesions integrate normally with (at least) $2^{\circ}$ spacing (Ciaramelli et al. 2007); (2) backward-masked contours spaced within $2^{\circ}$ can be integrated with only $30 \mathrm{~ms}$ of presentation, which is too brief for significant top-down modulation (Mandon and Kreiter 2005); and (3) visual agnosic patients with damaged occipital areas beyond V1 normally integrate closely spaced elements (Giersch et al. 2000). However, the relative contributions of intrinsic horizontal connections and feedback mechanisms to CI continue to be an active topic of investigation (De Meyer and Spratling 2009; Hess et al. 2003).

Persons with schizophrenia have been consistently shown to be poor at CI (Silverstein and Keane 2011a; Uhlhaas and Silverstein 2005). The deficit correlates with poor premorbid functioning, poor outcome, and disorganization (Silverstein and Keane 2011a); it is also associated with reduced BOLD signal response (fMRI) in areas V2-V4 (Silverstein et al. 2009a) - the same areas implicated in prior studies of integration in healthy humans and non-human primates (Kourtzi et al. 2003). But what is it about CI that causes problems in schizophrenia? One simple possibility is spacing-patients are simply not able to coordinate complex contextual interactions between segregated pieces of the proximal array and this inability becomes more apparent as those pieces are strewn further apart. Children exhibit this tendency. Kovács and colleagues showed that as Gabors of a target shape become more distantly spaced (from .9 to $1.7^{\circ}$ ), children (ages 5-6) became worse relative to adults (ages 19-35) in detecting an enclosed integrated surface. Such results imply that either (1) integrating more distantly spaced elements requires greater reliance on feedback connections (especially from V2) and these are not functioning in full capacity; or (2) integrating distantly spaced elements requires greater reliance on longer-range horizontal axonal connections, which again may be poorly developed or underutilized. Either of these possibilities may describe schizophrenia. The illness has been characterized as involving aberrant functional connectivity (Lynall et al. 2010), which would presumably be more apparent when coordinating more distant retinotopic areas. Moreover, visual dysfunction in schizophrenia has been attributed to compromised top-down visual feedback (van Assche and Giersch 2011; Gold et al. 2007), which again would imply worse CI performance at larger spacings.

To test the possibility of a reduced spatial range of integration in schizophrenia, we utilized a CI paradigm similar to that of Kovács et al. (1999). The target elements were spaced by .7 or $1.4^{\circ}$ of visual angle, and each spacing condition was presented at a variety of signal-tonoise ratios (see Fig. 1). If patient impairments become worse with larger target spacing, then that would imply either faulty feedback (Silverstein et al. 2006), dysfunctional longerrange lateral connectivity in V1/V2 (McGlashan and Hoffman 2000; Must et al. 2004) or both. On the other hand, if the level of patients' CI impairment does not vary as a function of interelement spacing - and thus if the impairment is largely independent of the stimulus- 
driven grouping strength between adjacent target elements-then that could imply a more general problem in representing a globally integrated shape.

\section{Methods}

Observers

The study examined 25 healthy controls and 36 persons with schizophrenia. Observers were recruited from five different sites: University of California-Davis, Maryland Psychiatric Research Center at the University of Maryland, University of Medicine and Dentistry of New Jersey, University of Minnesota-Twin Cities, and Washington University in St Louis. All were recruited from outpatient clinics, community centers, and local settings (e.g., grocery stores) via flyers and online advertisements. Monetary compensation was provided for participation. The study was approved by the Institutional Review Boards at all participating institutions; written informed consent was obtained from all subjects after explanation of the nature and possible consequences of the study. The research followed the tenets of the Declaration of Helsinki.

For both controls and schizophrenia patients, the inclusion/exclusion criteria included: (1) age 18-65 years; (2) no clinically significant head injury (loss of consciousness for $20 \mathrm{~min}$ or overnight hospitalization) or neurological disease; (3) no diagnosis of mental retardation or pervasive developmental disorder; (4) no substance dependence in the past 6 months and no substance abuse in the past month; (5) sufficient spoken English so as to be able to complete testing validity, (6) a score of six or higher on the Wechsler Test of Adult Reading (WTAR) as a measure of premorbid IQ (Wechsler 2001); (7) ability to give valid informed consent; and (8) passed alcohol and drug testing on each day of testing. Additional criteria for schizophrenia patients were the following: (1) Diagnostic and Statistical Manual of Mental Disorders, Fourth Edition (DSM-IV) diagnosis of schizophrenia or schizoaffective disorder (based on SCID interview, see below); (2) no medication changes in the prior month or anticipated in the upcoming month; and (3) stable outpatient or partial hospital status. Additional criteria for controls were the following: (1) no history of schizophrenia or any other psychotic disorder, including bipolar disorder; (2) no current major depression; and (3) no current psychotropic- or cognition-enhancing medication. All subjects reported normal or corrected-to-normal vision and were naïve to the purposes of the task. The final schizophrenia and control groups were matched at the group level for gender, age, race, and parental socioeconomic status SES, the last of which was measured using the Hollingshead Index (Hollingshead and Redlich 1958). Demographic and clinical information for participants is shown in Table 1.1

A masters-level clinician conducted or supervised diagnostic assessments using the Structured Clinical Interview for DSM-IV-Text Revision (First et al. 2002) and the 24-item Brief Psychiatric Rating Scale (Overall and Gorham 1962; Ventura et al. 1993a, b). Assessments of functional capacity included the following: the Brief version of the University of California, San Diego, Performance-based Skills Assessment (Patterson et al. 2001, UPSA-B); and the Specific Levels of Functioning Scale ("Participant" version; Schneider and Struening 1983, SLOF).

\section{Apparatus}

The apparatus was the same at each of five sites. The stimuli were presented on Samsung 2243BWX LCD monitors, which had a resolution of 1,680 × 1,050 pixels, a viewable screen

\footnotetext{
${ }^{1}$ For more information on strategies for recruitment, inclusion/exclusion criteria, clinical rater training, clinical assessment of participants, and general testing procedures, see Henderson et al. (2012).
} 
of 47.5 by $29.8 \mathrm{~cm}$, and a refresh rate of $60 \mathrm{~Hz}$. Subjects were positioned $61 \mathrm{~cm}$ from the screen. The stimuli were generated with a Dell computer (3.0 GHz, 6 M, 1,333 MHz FSB), and the task was implemented in E-prime 2.0. Displays were standardized across sites using Spyder 3.0 Elite. Standard overhead fluorescent lights were used with no additional lamp lighting.

\section{Stimulus and procedure}

Stimuli consisted of target Gabor elements on a square background $\left(13.3^{\circ}\right.$ on a side) that had a luminance of $23 \mathrm{~cd} / \mathrm{m}^{2}$. Each Gabor's Gaussian envelope size (SD) and wavelength was equal to $.2^{\circ}$. All Gabors were identical except for their positions and orientations. The number of Gabor elements composing the targets for the small and large gap conditions was 12 and 24, respectively. Target Gabors were separated by 0.7 or $1.4^{\circ}$ of visual angle for the small and large gap conditions, respectively. The distance between adjacent distractors divided by the distance between adjacent contour elements-which reflects the actual signal-to-noise ratio (SNR)—varied between .5 and .9 (in steps of .5). More simply, SNR was varied by adding or subtracting noise patches (with more noise patches making the task harder).

On each trial, the stimulus was presented for $3 \mathrm{~s}$ and subjects were told to try to respond during that period. Subjects pressed one of two keys to indicate whether the shape was leftward or rightward pointing. A black screen appeared immediately after the $3 \mathrm{~s}$ or subject response (whichever came first). Presentation of the different spacing and signal-to-noise ratio conditions was randomized. For each spacing condition, there were 9 SNRs, each of which incorporated eight unique trials. Thus, each subject completed 144 trials.

A practice session preceded the actual experiment and proceeded as follows. First, two leftand right-pointing demonstration stimuli (of each gap size) were sequentially shown on the screen until the subject reported seeing the oval shape. Next, subjects viewed a shape for $3 \mathrm{~s}$, and a luminance-defined black line was then drawn around that shape immediately after response or after $3 \mathrm{~s}$ (whichever came first). Afterward, 8 scored practice trials were given to further familiarize subjects with the task. If subjects did not respond correctly (and within the time limit) in at least 5 out of 8 of these trials, another block of practice trials was given. Up to 5 practice blocks ( 40 trials) were given. Only 1 control and 3 patients needed more than 1 practice block. The two groups were undifferentiated in the number of practice trials received $(p=.49)$.

\section{Data analysis}

Prior to carrying out all analyses, every other timed-out trial was coded as a correct response, so that chance performance would be $50 \%$ regardless of whether a subject chose to guess or time-out on a trial. We next calculated the total percent correct at each SNR for each subject and spacing condition. We removed 4 subjects ( 3 patients) whose overall proportion correct was less than or equal to $50 \%$ on the task. For each subject and spacing

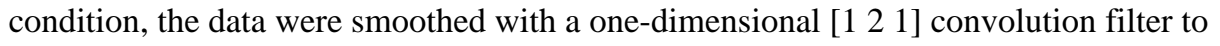
decrease volatility between adjacent SNR accuracy values (and to improve the curve fitting process). The two outer SNR values (50 and 90) could not be assigned a weighted average in the convolution process and thus were not used in the subsequent analyses. These smoothed accuracy data were fit with a cumulative Weibull (Wichmann and Hill 2001) function:

$$
\Psi(x ; \alpha, \beta, \gamma, \lambda)=\gamma+(1-\gamma-\lambda)\left(1-\exp \left(-(x / \alpha)^{\beta}\right)\right)
$$


$\Psi$ corresponds to the predicted probability correct, $x$ denotes the SNR; $a$ and $\beta$ determine the threshold and slope of the psychometric function; $\gamma$ is the guessing rate and was fixed at .5; and $\lambda$ refers to the upper asymptote of the fitted curve and traditionally corresponds to the stimulus- independent error rate. A threshold for a Weibull curve corresponds to the SNR needed to produce $81.6 \%$ accuracy; this accuracy percentage is lower if the subject has a positive $\lambda$ value. Data for one control could not be fit with a curve for either spacing condition; data for one patient could not be fit with a curve for the large spacing condition only. (Parameter estimates for these subjects did not converge because the proportion correct did not improve with increased SNR.) The proportions of excluded patients (4/36) and controls $(2 / 25)$ did not differ significantly (Fisher's Exact Test, one-sided $=.523)$. Finally, we compared the $a$ and $\beta$ parameter estimates with a 2 (gap size) $\times 2$ (subject group) mixedmodel ANOVA. The thresholds were log-transformed (base 10) to be rendered approximately normal. The a parameters were compared directly since a corresponds to different percentages correct at different lapse rates (Kingdom and Prins 2009) The $\lambda$ parameters were not analyzed because they are unreliable unless a large number of trials are collected at the easiest difficulty levels. (The purpose of including $\lambda$ as a free parameter is mainly to improve the estimates of $\alpha$ and $\beta$; (Wichmann and Hill 2001; Kingdom and Prins 2009.)

\section{Results}

Results are depicted in Fig. 2. The curves fit the accuracy data well for controls (median Rsquared $>.92$ for each condition) and were acceptable for patients (median R-squared $>.82$ for each condition). For the $\beta$ analysis, there were no main effects or interactions (all $p \mathrm{~s}>$. $10)$. For the threshold (a) analysis, patients had lower thresholds than controls overall $\left(F(1,53)=6.8, p=.01, \eta_{p}^{2}=.114\right)$ and the large spacing stimuli were more difficult to discriminate than the smaller spaced stimuli, $F(1,53)=11.7, p=.001, \eta_{p}^{2}=.181$. Most importantly, increasing the spacing did not make patients perform worse relative to controls, $\left.F(1,53)=.37, p=.55, \eta_{p}^{2}=.007\right)$. These results were robust and remained, for example, if the fitted parameters had different boundary conditions, if two outlier values (both patients) were removed, if a cumulative logistic function were fit to the same data, or if the data were unsmoothed. To be sure that the lack of interaction did not change with the kind of analysis performed, we also examined the integral of the distribution functions and the total percent correct across SNR. Each of these two analyses yielded between-group differences ( $p$ s $<$. 02 ), a main effect of gap size ( $p s<.001)$, but no interaction ( $p$ s > .37). Furthermore, there were no correlations among patients between symptomatology (scores of each BPRS category, see Table 1) and log threshold for either the small or large spacing condition (all $p s>.12$ ), which is not surprising being that the patient group was largely asymptomatic. Threshold and medication dosage did not significantly correlate for the small spacing condition ( $p=.07$, Spearman's $\rho=.34)$, the large spacing condition $(p=.10, \rho=.31)$, or the threshold difference $(p=.47, \rho=.14)$. The same three threshold values did not correlate with premorbid IQ (as measured by the WTAR) for patients alone, or for all subjects (all $p \mathrm{~s}$ $>$.13).

\section{Discussion}

Our results replicate numerous past studies (Kozma-Weibe et al. 2006; Silverstein et al. 2009b; for a review, see Silverstein and Keane 2011a) in demonstrating that patients are not as adept at integrating and combining elements to represent global shape. Moreover, performance worsened for both groups as target elements spacing increased. Finally, and most importantly, even when the target spacing was doubled to $1.4^{\circ}$ of visual angle-and thus even when subjects needed to rely more on later-developing mechanisms of long-range 
lateral connectivity and top-down feedback — the magnitude of patient deficits did not increase.

\section{Explaining the effects of gap size and patient group}

The advantage of smaller spacing was expected on the ground that physically specifying a larger portion of a contour - that is, increasing a contour's "support ratio"-increases the precision with which that contour is represented (Hadad et al. 2010; Pillow and Rubin 2002; Shipley and Kellman 1992). It is important to note, however, that Kovács et al. (1999) and others did not find a deleterious effect of target spacing on adult performance (Braun 1999). The discrepancy can be reconciled by the fact that these studies required only contour detection, while the present study required shape discrimination. Whereas efficiently detecting an integrated shape can occur even when the enclosing contour is poorly specified (Pillow and Rubin 2002), discrimination of similarly appearing alternatives requires relatively precise edge representation.

An objection is that subjects did not necessarily have to integrate a whole shape to perform the task; they could have just integrated only a diagnostic part of the shape (Mandon and Kreiter 2005). This possibility is not problematic because subjects would still need to integrate multiple patches in both distance conditions, and the data provide no evidence that the larger spacings were especially difficult for patients.

Can generalized deficits in motivation or visual acuity explain our results? We deem it unlikely. In a study with the same exclusion/inclusion criteria and the same recruitment sites as the present, controls $(N=132)$ had an average logMAR acuity of $.114(20 / 26)$ and patients $(N=104)$ had an average acuity of $.168(20 / 29)$ (Strauss et al. in preparation). Such differences would doubtfully be significant in a study of our size. Moreover, as can be guessed from Fig. 2, there was no group difference on the three easiest SNRs of the small spacing condition $(p>.6)$, indicating that patients were properly understanding the task and seeing the Gabor stimuli. In addition, the three-parameter curve fitting procedure takes into account attentional errors ("lapsing errors") by essentially making it easier for a subject to reach threshold as the lapse rate increases (Wichmann and Hill 2001). Lastly and most importantly, even if generalized deficits altered patient behavior, there is no reason to think that such deficits would suppress an otherwise significant interaction between spacing and subject group.

The lack of interaction between gap size and subject group implies that cortical mechanisms that combine information over larger distal ranges are not differentially impaired in schizophrenia. That is, the longer-range faciliatory interactions between orientation-tuned spatial frequency channels in V1/V2 and the low-level feedback mechanisms that span larger cortical areas do not play a special role in patients' CI dysfunction.

The lack of interaction leaves open an interesting possibility, viz., that the patient deficits in the CI task are independent of low-level linking. In other words, it is at least possible that poor performance owes not to local element linking but to a higher-level inability to form a global shape representation from the individually connected elements. There is some evidence for this view. When four sectored circles form a single "Kanizsa" (illusory) shape, people with schizophrenia show a normal sensitivity to perturbances of the filled-in path, but a poor ability to discriminate the overall shape (Keane et al. 2012b). In an EEG study, when schizophrenia patients attempted to detect a Kanizsa shape, there was an intact early ventral stream response (106-194 ms) for contour filling-in but a subsequent abnormal frontal response (240-400 ms) (Foxe et al. 2005). A related EEG study showed that-when attempting to name objects depicted by line drawings-patients were more adversely affected by fragmentation of the drawings than controls, and this effect corresponded to a 
differential neural response at relatively late stages in processing (Doniger et al. 2002). Other studies have also dissociated local contour interpolation from global shape perception (Giersch et al. 2000; Keane et al. 2012a). More generally, it has been supposed that the latest stages in object perception are those that are most acutely affected (Uhlhaas and Silverstein 2005), perhaps as a result of hypofunctioning NMDA glutamate receptors (Phillips and Silverstein 2003). Of course, the foregoing is not meant to imply that all high-level abilities are compromised in schizophrenia. Patients can suppress or enhance selected input (Luck and Gold 2008) or adapt to certain regularities in interelement grouping (Giersch and Rhein 2008). Future studies will need to test CI of closed and open contours (Kovács and Julesz 1993) to see whether CI deficits can be explained in terms of global shape processing (or "closure") deficits.

\section{Limitations and future directions}

Conclusions drawn from our data must be qualified in several important ways. One is that because all subjects were highly asymptomatic and clinically stable (see Table 1), the results may not generalize to other segments of the schizophrenia population. In particular, there remains the possibility that more disorganized patients would exhibit a differential deficit at larger element spacings, since they are the most susceptible to perceptual organization impairments (Phillips and Silverstein 2003; Uhlhaas and Silverstein 2005; Silverstein and Keane 2011a; Silverstein et al. 2000). Relatedly, more severely disabled patients display posteriorization of gray matter loss in the occipital lobe (Mitelman and Buchsbaum 2007), and the laterdeveloping low-level circuitry in these individuals may be especially compromised as Gabors are distributed over larger spatial gaps. Another limitation is that all patients in our study were on medication at the time of testing. While medication effects cannot be ruled out, we do not regard them as particularly troubling because: (1) our results did not correlate with dosage; (2) other visual integration studies failed to find dosage correlations (Foxe et al. 2005; Doniger et al. 2002; Silverstein et al. 2009b); and (3) unmedicated patients with at least a 3-week washout period exhibit visual integration dysfunction (Must et al. 2004).

A final noteworthy limitation is that our spacing conditions were restricted to .7 and $1.4^{\circ}$ of visual angle. Even though similar spacings caused children and adult performance to diverge in an detection study (Kovács et al. 1999), the large spacing value may not have been sufficient to produce patient differences. For example, persons with frontal lobe injuries integrate normally at $2^{\circ}$, but not at 3 or $4^{\circ}$ (Ciaramelli et al. 2007). Considering the welldocumented frontal cortex abnormalities in schizophrenia (Volk and Lewis 2010), increasing the gap size past $2^{\circ}$ may well produce the interaction of interest. Importantly, if such large spacings were necessary, the implicated visual pathway in schizophrenia would be qualitatively different from that considered in the present study, involving much later stages in processing, more susceptibility to attention and memory, and little dependency on connectivity in primary visual cortex (Loffler 2008), p. 2114). The result, if obtained, would reveal more about the ability to generate and deploy cognitive strategies than it would about the capacity to reflexively unify elements in occipital cortex, which was the goal of the present study. In sum, our results provide evidence that higher-functioning patients with schizophrenia have a relatively constant level of CI dysfunction for elements spaced within $1.5^{\circ}$. This indicates that, in schizophrenia, the mechanisms of low-level feedback and lateral connectivity that develop in middle childhood and early adolescence are no more impaired relative to those that mature by age 5 or 6 .

\section{Acknowledgments}

We would like to thank the following Research Assistants for helping to bring this project to completion: Robin S. Lyons, Yushi Wang, Jamie Joseph (UMDNJ); Emily Thomason, Cindy Feldt, Jennifer Shuite, Melissa Cornejo 
(Washington University, St. Louis); Sharon August, Leeka Hubzin, Samual Kaiser, Tatyanna Matveeva (MPRC); Dori Henderson, Madelyn Steen, Anna Schnurrer, Joe Lowinske, Lindsay Swanson (Minnesota); and Brittaney Haley (UC-Davis). We also appreciate the input of four anonymous reviewers whose extensive comments improved the manuscript. Funding for this research was provided by the following collaborative RO1s: MH084828-01 to SMS (UMDNJ), MH84840 to DMB (Washington University, St. Louis), MH084826 to CSC and JDR (UC Davis), MH084821 to JG (MPRC), and MH084861 to AM (Minnesota). Support from an F32 (MH094102-01A1) was provided to BPK (UMDNJ/Rutgers) during the write-up of the manuscript.

\section{References}

Angelucci A, Levitt JB, Walton EJ, Hupe JM, Bullier J, Lund JS. Circuits for local and global signal integration in primary visual cortex. J Neurosci. 2002; 22(19):8633-8646. [PubMed: 12351737]

Braun J. On the detection of salient contours. Spat Vis. 1999; 12(2):211-225. [PubMed: 10221428]

Chandna A, Pennefather PM, Kovács I, Norcia AM. Contour integration deficits in anisometropic amblyopia. Invest Ophthalmol Vis Sci. 2001; 42(3):875-878. [PubMed: 11222553]

Ciaramelli E, Leo F, Del Viva MM, Burr DC, Ladavas E. The contribution of prefrontal cortex to global perception. Exp Brain Res. 2007; 181(3):427-434. [PubMed: 17401551]

De Meyer K, Spratling MW. A model of non-linear interactions between cortical top-down and horizontal connections explains the attentional gating of collinear facilitation. Vision Res. 2009; 49(5):553-568. [PubMed: 19162060]

Del Viva MM, Agostini R. Visual spatial integration in the elderly. Invest Ophthalmol Vis Sci. 2007; 48(6):2940-2946. [PubMed: 17525231]

Doniger GM, Foxe JJ, Murray MM, Higgins BA, Javitt DC. Impaired visual object recognition and dorsal/ventral stream interaction in schizophrenia. Arch Gen Psychiatry. 2002; 59(11):1011-1020. [PubMed: 12418934]

Dresp B, Grossberg S. Contour integration across polarities and spatial gaps: from local contrast filtering to global grouping. Vis Res. 1997; 37(7):913-924. [PubMed: 9156188]

Field DJ, Hayes A, Hess RF. Contour integration by the human visual system: evidence for a local "association field". Vis Res. 1993; 33(2):173-193. [PubMed: 8447091]

First, MB.; Spitzer, RL.; Miriam, G.; Williams, JBW. Structured clinical interview for DSM-IV-TR Axis I disorders, research version, non-patient edition (SCID-I/NP). New York, NY: Biometrics Research, New York State Psychiatric Institute; 2002.

Foxe JJ, Murray MM, Javitt DC. Filling-in in schizophrenia: a high-density electrical mapping and source-analysis investigation of illusory contour processing. Cereb Cortex. 2005; 15(12):19141927. [PubMed: 15772373]

Giersch A, Rhein V. Lack of flexibility in visual grouping in patients with schizophrenia. J Abnorm Psychol. 2008; 117(1):132-142. [PubMed: 18266491]

Giersch A, Humphreys G, Boucart M, Kovács I. The computation of occluded contours in visual agnosia: evidence for early computation prior to shape binding and figure-ground coding. Cogn Neuropsychol. 2000; 17(8):731-759. [PubMed: 20945203]

Gold JM, Fuller RL, Robinson BM, Braun EL, Luck SJ. Impaired top-down control of visual search in schizophrenia. Schizophr Res. 2007; 94(1-3):148-155. [PubMed: 17544632]

Green MF, Lee J, Wynn JK, Mathis KI. Visual masking in schizophrenia: overview and theoretical implications. Schizophr Bull. 2011; 37(4):700-708. [PubMed: 21606322]

Grossberg S, Raizada R. Contrast-sensitive perceptual grouping and object-based attention in the laminar circuits of primary visual cortex. Vis Res. 2000; 40(10-12):1413-1432. [PubMed: 10788649]

Hadad B-S, Maurer D, Lewis TL. The development of contour interpolation: evidence from subjective contours. J Exp Child Psychol. 2010; 106(2-3):163-176. [PubMed: 20227089]

Henderson D, Poppe AB, Barch DM, Carter CS, Gold JM, Ragland JD, Silverstein SM, Strauss ME, MacDonald AW III. Optimization of a goal maintenance task for use in clinical applications. Schizophr Bull. 2012; 38(1):104-113. [PubMed: 22199092]

Hess RF, Hayes A, Field DJ. Contour integration and cortical processing. J Physiol Paris. 2003; 97(23):105-119. [PubMed: 14766137]

Hollingshead, AD.; Redlich, FC. Social class and mental illness. New York: Wiley; 1958. 
Javitt DC. Sensory processing in schizophrenia: neither simple nor intact. Schizophr Bull. 2009; 35(6): 1059-1064. [PubMed: 19833806]

Kapadia MK, Ito M, Gilbert CD, Westheimer G. Improvement in visual sensitivity by changes in local context: parallel studies in human observers and in V1 of alert monkeys. Neuron. 1995; 15(4): 843-856. [PubMed: 7576633]

Keane BP, Lu H, Papathomas TV, Silverstein SM, Kellman PJ. Is interpolation cognitively encapsulated? Measuring the effects of belief on Kanizsa shape discrimination and illusory contour formation. Cognition. 2012a

Keane, BP.; Mikkilineni, D.; Papathomas, TV.; Silverstein, SM. Impaired shape integration but normal illusory contour formation in schizophrenia: evidence for a high level grouping deficit. Poster at Vision Sciences Society Conference, May; Naples, FL. 2012b. p. 404-418.

Kingdom, FAA.; Prins, N. Psychophysics: a practical introduction. New York: Academic Press; 2009.

Knight RA, Silverstein SM. A process-oriented approach for averting confounds resulting from general performance deficiencies in schizophrenia. J Abnorm Psychol. 2001; 110(1):15-30. [PubMed: 11261389]

Kourtzi Z, Tolias AS, Altmann CF, Augath M, Logothetis NK. Integration of local features into global shapes: monkey and human FMRI studies. Neuron. 2003; 37(2):333-346. [PubMed: 12546827]

Kovács I, Julesz B. A closed curve is much more than an incomplete one: effect of closure in figureground segmentation. Proc Natl Acad Sci USA. 1993; 90(16):7495-7497. [PubMed: 8356044]

Kovács I, Kozma P, Fehér A, Benedek G. Late maturation of visual spatial integration in humans. Proc Natl Acad Sci USA. 1999; 96(21):12204-12209. [PubMed: 10518600]

Kozma-Weibe P, Silverstein SM, Fehér A, Kovács I, Uhlhaas P, Wilkniss SM. Development of a world-wide web based contour integration test. Comput Hum Behav. 2006; 22:971-980.

Li W, Gilbert CD. Global contour saliency and local colinear interactions. J Neurophysiol. 2002; 88(5):2846-2856. [PubMed: 12424317]

Li W, Piëch V, Gilbert CD. Contour saliency in primary visual cortex. Neuron. 2006; 50(6):951-962. [PubMed: 16772175]

Loffler G. Perception of contours and shapes: low and intermediate stage mechanisms. Vis Res. 2008; 48(20):2106-2127. [PubMed: 18502467]

Luck SJ, Gold JM. The construct of attention in schizophrenia. Biol Psychiatry. 2008; 64(1):34-39. [PubMed: 18374901]

Lynall ME, Bassett DS, Kerwin R, McKenna PJ, Kitzbichler M, Muller U, Bullmore E. Functional connectivity and brain networks in schizophrenia. J Neurosci. 2010; 30(28):9477-9487. [PubMed: 20631176]

Mandon S, Kreiter AK. Rapid contour integration in macaque monkeys. Vis Res. 2005; 45(3):291300. [PubMed: 15607346]

McGlashan TH, Hoffman RE. Schizophrenia as a disorder of developmentally reduced synaptic connectivity. Arch Gen Psychiatry. 2000; 57(7):637-648. [PubMed: 10891034]

Mitelman SA, Buchsbaum MS. Very poor outcome schizophrenia: clinical and neuroimaging aspects. Int Rev Psychiatry. 2007; 19(4):345-357. [PubMed: 17671868]

Must A, Janka Z, Benedek G, Kéri S. Reduced facilitation effect of collinear flankers on contrast detection reveals impaired lateral connectivity in the visual cortex of schizophrenia patients. Neurosci Lett. 2004; 357(2):131-134. [PubMed: 15036592]

Overall JE, Gorham DR. The brief psychiatric rating scale. Psychol Rep. 1962; 10:799.

Patterson TL, Goldman S, McKibbin CL, Hughs T, Jeste DV. UCSD performance-based skills assessment: development of a new measure of everyday functioning for severely mentally ill adults. Schizophr Bull. 2001; 27(2):235-245. [PubMed: 11354591]

Phillips WA, Silverstein SM. Convergence of biological and psychological perspectives on cognitive coordination in schizophrenia. Behav Brain Sci. 2003; 26(1):65-82. discussion 82-137. [PubMed: 14598440]

Pillow J, Rubin N. Perceptual completion across the vertical meridian and the role of early visual cortex. Neuron. 2002; 33(5):805-813. [PubMed: 11879656] 
Place EJ, Gilmore GC. Perceptual organization in schizophrenia. J Abnorm Psychol. 1980; 89(3):409_ 418. [PubMed: 7410708]

Schneider LC, Struening EL. SLOF: a behavioral rating scale for assessing the mentally ill. Soc Work Res Abstr. 1983; 19:9-21. [PubMed: 10264257]

Shipley TF, Kellman PJ. Strength of visual interpolation depends on the ratio of physically specified to total edge length. Percept Psychophys. 1992; 52(1):97-106. [PubMed: 1635860]

Silverstein SM, Keane BP. Perceptual organization impairment in schizophrenia and associated brain mechanisms: review of research from 2005 to 2010. Schizophr Bull. 2011a; 37(4):690-699. [PubMed: 21700589]

Silverstein SM, Keane BP. Vision science and schizophrenia research: toward a re-view of the disorder editors' introduction to special section. Schizophr Bull. 2011b; 37(4):681-689. [PubMed: 21700588]

Silverstein SM, Knight RA, Schwarzkopf SB, West LL, Osborn LM, Kamin D. Stimulus configuration and context effects in perceptual organization in schizophrenia. J Abnorm Psychol. 1996; 105(3): 410-420. [PubMed: 8772011]

Silverstein SM, Kovacs I, Corry R, Valone C. Perceptual organization, the disorganization syndrome, and context processing in chronic schizophrenia. Schizophr Res. 2000; 43(1):11-20. [PubMed: 10828411]

Silverstein SM, Hatashita-Wong M, Schenkel LS, Wilkniss S, Kovacs I, Feher A, Smith T, Goicochea C, Uhlhaas P, Carpiniello K, Savitz A. Reduced top-down influences in contour detection in schizophrenia. Cogn Neuropsychiatry. 2006; 11(2):112-132. [PubMed: 16537237]

Silverstein SM, Berten S, Essex B, Kovacs I, Susmaras T, Little DM. An fMRI examination of visual integration in schizophrenia. J Integr Neurosci. 2009; 8(2):175-202. [PubMed: 19618486]

Stettler DD, Das A, Bennett J, Gilbert CD. Lateral connectivity and contextual interactions in macaque primary visual cortex. Neuron. 2002; 36(4):739-750. [PubMed: 12441061]

Strauss ME, Barch DM, Carter CS, Keane BP, Gold JM, Ragland JD, Silverstein SM, MacDonald AW III. Test-retest reliability for cognitive psychometric tests in schizophrenia. (in preparation).

Uhlhaas PJ, Silverstein SM. Perceptual organization in schizophrenia spectrum disorders: empirical research and theoretical implications. Psychol Bull. 2005; 131(4):618-632. [PubMed: 16060805]

Uhlhaas PJ, Phillips WA, Mitchell G, Silverstein SM. Perceptual grouping in disorganized schizophrenia. Psychiatry Res. 2006; 145(2-3):105-117. [PubMed: 17081620]

van Assche M, Giersch A. Visual organization processes in schizophrenia. Schizophr Bull. 2011; 37(2):394-404. [PubMed: 19703942]

Ventura J, Green MF, Shaner A, Liberman RP. Training and quality assurance on the Brief Psychiatric Rating Scale: the “drift busters”. Int J Methods Psychiatric Res. 1993a; 3:221-226.

Ventura J, Lukoff D, Nuechterlein KH, Liberman RP, Green MF, Shaner A. Brief Psychiatric Rating Scale (BPRS) expanded version: scales, anchor points, and administration manual. Int J Methods Psychiatric Res. 1993b; 3:227-243.

Volk DW, Lewis DA. Prefrontal cortical circuits in schizophrenia. Curr Top Behav Neurosci. 2010; 4:485-508. [PubMed: 21312410]

Wechsler, D. Wechsler test of adult reading. San Antonio: The Psychological Corporation; 2001.

Wichmann F, Hill N. The psychometric function: I. Fitting, sampling, and goodness of fit. Percept Psychophys. 2001; 63(8):1293-1313. [PubMed: 11800458]

Zhang NR, von der Heydt R. Analysis of the context integration mechanisms underlying figure-ground organization in the visual cortex. J Neurosci. 2010; 30(19):6482-6496. [PubMed: 20463212] 


\section{Signal to Noise Ratio (SNR)}

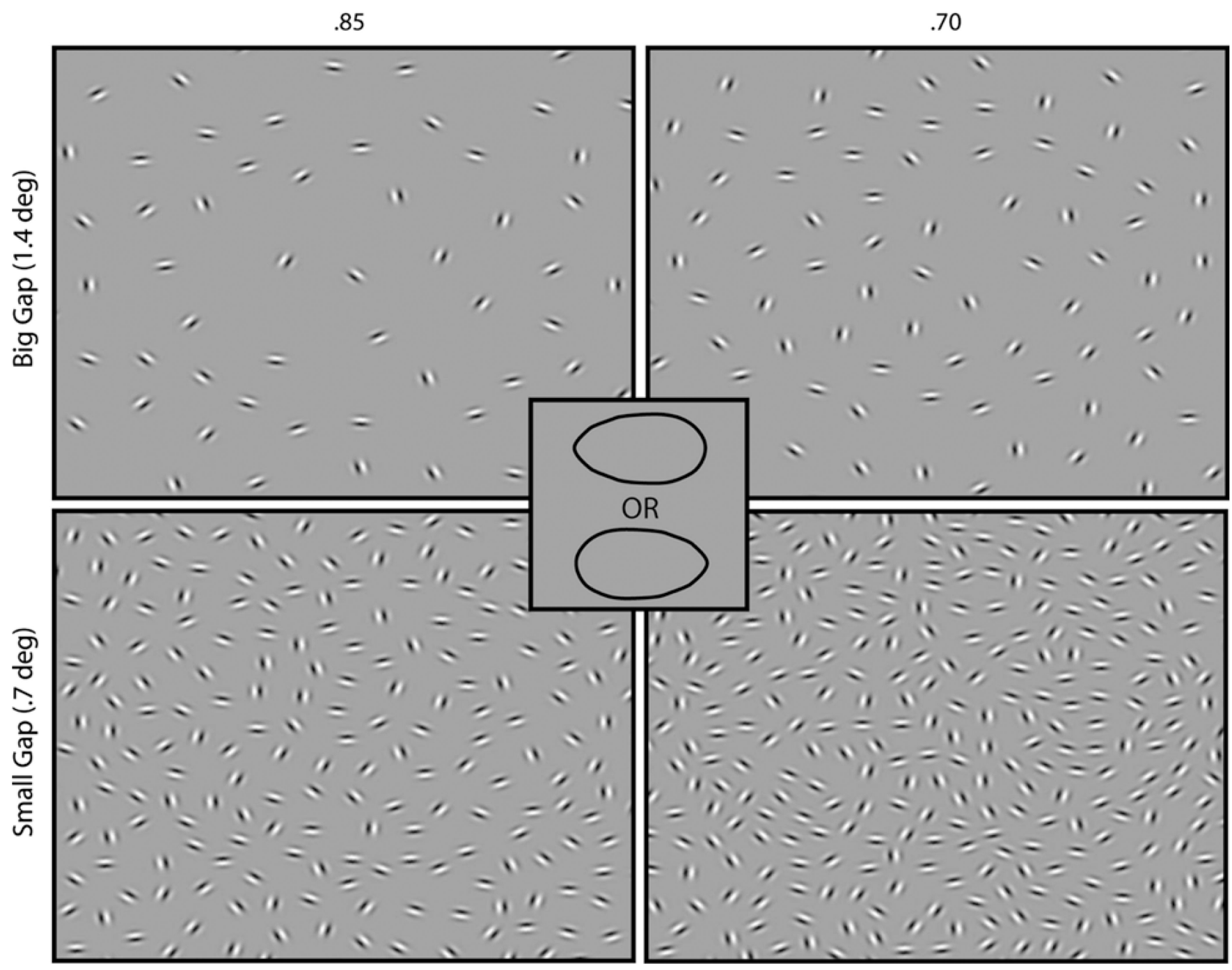

Fig. 1.

Stimuli and discriminated alternatives. Contour integration displays (magnified and cropped for illustration) are shown for two SNRs of each spacing condition. The SNR was determined by the number of noise patches accompanying the target. On each trial, subjects had to determine whether the embedded ovular target was pointing to the left or right 

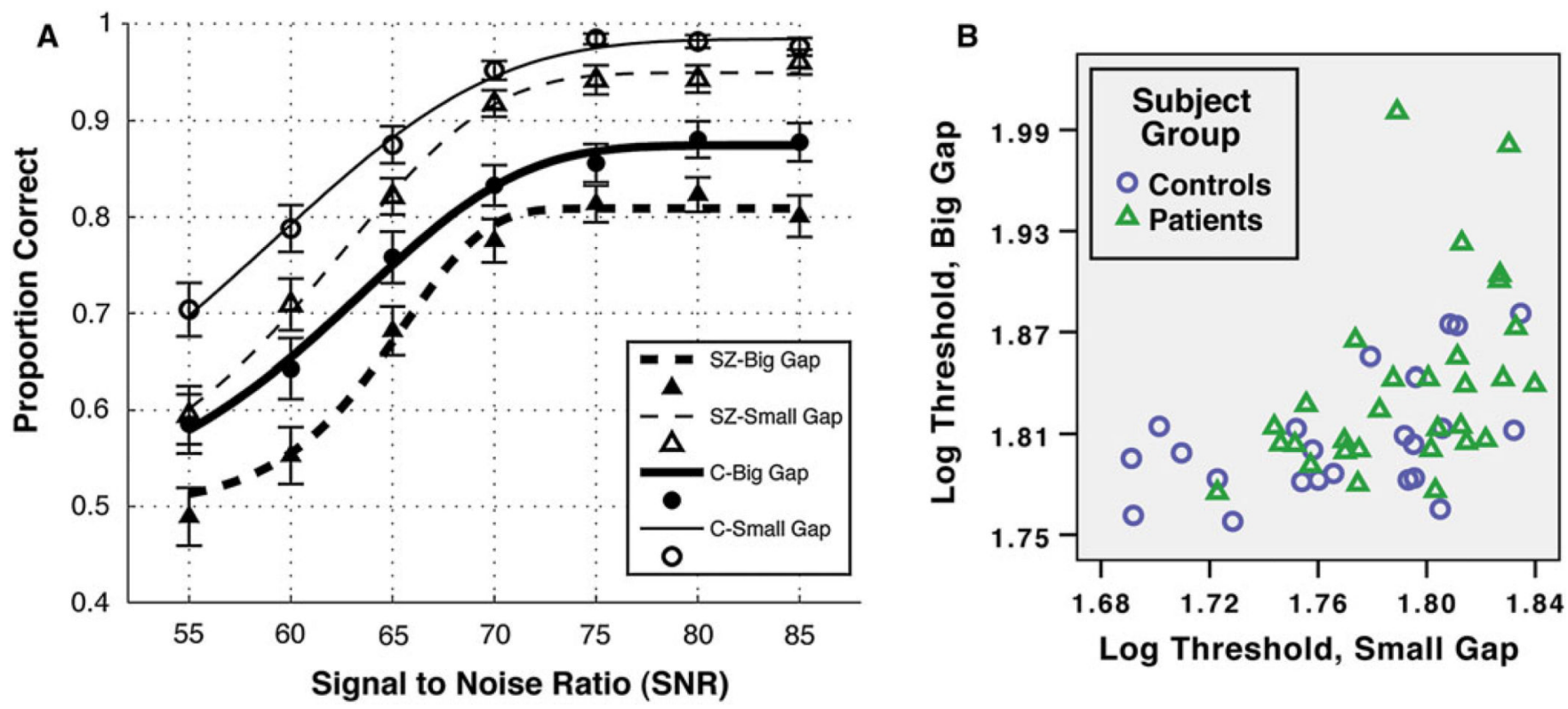

Fig. 2.

Results. a Average accuracy and fitted cumulative Weibull curves are shown for patients $(\mathrm{SZ})$ and controls $(\mathrm{C})$ for two spacing conditions $\left(\mathrm{Big}=1.4^{\circ} ;\right.$ Small $\left.=.7^{\circ}\right)$. Black circles and squares are for patients and controls, respectively. Open and filled symbols are for small and large gap conditions, respectively. Errors show \pm SEM. Subjects who could not be fit with a curve or whose accuracy was below chance were not included in the graph data. b Colored symbols depict variability of log thresholds for each group and spacing condition (with two outliers removed). As shown, patients performed worse than controls for each spacing condition, and this difference did not significantly change as the spacing increased from .7 to 1.4 degrees 


\section{Table 1}

\section{Demographic and symptom characteristics of study sample}

Averages (and SDs) are shown, except where indicated.SZ, schizophrenia or schizoaffective disorder; $S E S$, socioeconomic status as measured with the Barratt Simplified Measure of Social Status based on the Hollingshead Index. SLOF, Specific Levels of Functioning Scale; UPSA-B, UCSD performance-based skills assessment-brief; $B P R S$, Brief Psychiatric Rating Scale, BPRS values denote average scores per item for each scale and range from 1 to 7 (with 1 indicating no symptoms). Medication information was missing for two patients. A chisquare test compared gender and ethnic composition; all other $p$ values derived from uncorrected $t$ tests

\begin{tabular}{|c|c|c|c|}
\hline Variables & $\mathrm{SZ}(N=36)$ & Healthy controls $(N=25)$ & $p$ value \\
\hline Age & $38(11)$ & $41(12)$ & .38 \\
\hline Education, self & $13.6(2.4)$ & $14.9(1.8)$ & .006 \\
\hline Education, father & $14.1(3.4)$ & $11.5(2.5)$ & .003 \\
\hline Education, mother & $12.7(2.7)$ & $12.4(2.5)$ & .63 \\
\hline Gender, $\%$ male & 60 & 52 & .54 \\
\hline SES, parental & $44.5(13.5)$ & $40.3(12.0)$ & .22 \\
\hline SES, participant & $28.0(9.3)$ & $34.2(9.0)$ & .01 \\
\hline SLOF, total & $4.2(.3)$ & $4.8(.15)$ & $<.001$ \\
\hline UPSA-B, total & $78.8(14.5)$ & $84.4(8.6)$ & .07 \\
\hline WTAR & $35.5(10.9)$ & $36.4(8.6)$ & .72 \\
\hline$\%$ Caucasian & 68.6 & 48 & .07 \\
\hline BPRS positive & $2.3(1.2)$ & NA & \\
\hline BPRS disorganized & $1.4(.6)$ & NA & \\
\hline BPRS negative & $1.8(.8)$ & NA & \\
\hline BPRS mania & $1.2(.5)$ & NA & \\
\hline BPRS depressed & $2.1(.9)$ & NA & \\
\hline Chlorpromazine, equivalent (mg/day) & $457(374)$ & NA & \\
\hline Neuroleptics: typical/atypical/both & $2 / 28 / 4$ & NA & \\
\hline Schizophrenia/schizoaffective & $25 / 11$ & NA & \\
\hline
\end{tabular}

